\title{
Nature Inspired Solutions for Green Energy
}

\author{
Jae Hoo Cho', Dong Hwa Kim², Ajith Abraham ${ }^{3}$ \\ ${ }^{1}$ Chungbuk National University, Korea \\ ${ }^{2}$ Hanbat National University, San 16-1, Duck Myoung-Dong, Yuseong-Gu, Daejeon, Korea \\ ${ }^{2}$ Machine Intelligence Research Labs (MIR Labs), Scientific Network for Innovation and Research \\ Excellence (SNIRE), USA
}

\begin{abstract}
Traditionally intelligence systems are popular in robotics, decision-making, multimedia processing, data mining etc. Recently, green technology and $\mathrm{CO}_{2}$ gas reduction issues have been a major issue, paving the way for artificial intelligence researchers to formulate effective solutions. Therefore, there are good prospects for researchers to study green technology and $\mathrm{CO}_{2}$ gas reduction problems because those issues are directly affecting our daily life. Energy saving and development, power management for renewable energy sources are very important for green technology and $\mathrm{CO}_{2}$ gas reduction. This paper describes our application methods studied up to now for evaluating green technology.
\end{abstract}

\section{Introduction}

With the rising energy costs and the threat of global warming, many business organizations are now recognizing the benefits of using green technology to reduce their carbon footprint and to minimize waste.

The green technology should provide tangible benefits to all businesses interested in reducing their impact on the environment. Hence we have to find green technology for industrial methods and to replace current technologies with green technology.

Especially, every year, energy consumption increases and more $\mathrm{CO}_{2}$ gases are emitted into the atmosphere. By 2020, one-third of the world's population may lack access to clean water, air, and energy. At the same time, it's estimated that energy demands will have further increased. By this situation, next generation will inherit a world that looks vastly different than today's. The environmental impacts of the today's fossil-fueldependent economy should be considered today's energy sources and operating method. We should decide about the best way to increase quantities of energy hidden under the world's energy system, which could yield far more energy than the traditional energy operating system or earth's remaining reserves of fossil fuel. We should also develop methods to increase energy efficiency. We have to develop new ways to more efficiently utilize today's energy sources through the development of energy-saving methods. Micro-model is one of these approaches to save energy.

In this paper, we illustrate the deployment of some artificial intelligence methods for optimal operation. We apply bacterial foraging optimization (BFO) method for energy system operation [1-4]. On the other hand, as natural selection tends to eliminate animals with poor foraging strategies through methods for locating, handling, and ingesting food and favor the propagation of genes of those animals that have successful foraging strategies. The BFO mimicking biological bacterial food-searching behavior has been applied in the field of optimization. This paper presents various approaches such as GAPSO (Genetic Algorithm - Particle Swarm Optimization) and GA-BF (Genetic AlgorithmBacterial Foraging) for some optimization problems related to green technology.

\section{Particle Swarm Optimization}

A population of particles is initialized with random positions $\vec{k}_{i}$ and velocity $\vec{v}_{i}$, and a function, $f$, is evaluated, using the particle's positional coordinates as input values. Positions and velocities are adjusted, and the function is evaluated with the new coordinates at each time-step. When a particle discovers a pattern that is better than any it has found previously, it stores the coordinates in a vector pbest ${ }_{j}$. The difference between pbest ${ }_{j}$ (the best point found by $i$ so far) and the individual's current position gbest is stochastically added to the current velocity, causing the trajectory to oscillate around that point. Further, each particle is defined within the context of a topological neighborhood comprising itself and some other particles in the population. The stochastically weighted difference between the neighborhood's best position gbest and the individual's current position is also added to its velocity, adjusting it for the next time-step. These adjustments to the particle's movement through the space cause it to search around the two best positions.

We used the position and velocity vector of PSO as follows: 


$$
\begin{aligned}
& v_{f, g}^{(t+1)}=w \cdot v_{j}^{(t)}+c_{1}^{*} \operatorname{Rand}_{()^{*}\left(\text { pbest }_{j, g}-k_{j, g}^{(t)}\right)} \\
& \left.+c_{2}^{*} \operatorname{Rand}^{*}\right)^{*}\left(\text { gbest }_{g}-k_{j, g}^{(t)}\right) \\
& \quad j=1,2, \ldots, n . \\
& \quad g=1,2, \ldots, m . \\
& \quad k_{j, g}^{(t+1)}=k_{j, g}^{(t)}+v_{j, g}^{(t+1)}, \quad k_{g}^{\min } \leq k_{j, g}^{(t+1)} \leq k_{g}^{\max }
\end{aligned}
$$

$n:$ The number of agent in each group

$m$ : The number of member in each group

$t$ : Number of reproduction steps

$v_{j, g}^{(t)}$

: The velocity vector of agent $j$ in reproduction

step of $t^{\text {th }} \quad V_{g}^{\min } \leq v_{j, g}^{(t)} \leq V_{g}^{\max }$

$k_{j, g}^{(t)}$

: The position vector of agent $j$ in reproduction

step of $t^{\text {th }}$.

$w$ : Weighting factor

$c 1, c 2$ : Acceleration constant

rand (), Rand (): Random value between 0 and 1

pbest $_{j}{ }_{j}$ : Optimal position vector of agent $j$

gbest : Optimal position vector of the group of agents

The variables $c_{1}^{*}$ Rand ()$^{*}$ and $c_{2}^{*}$ Rand ()$^{*}$ are random positive numbers, drawn from a uniform distribution and defined by an upper limit $c_{2}^{*}$ Rand ()$_{\max }^{*}$ which is a parameter of the system. In this version, the term variable $w \cdot v_{j}^{(t)}$ is limited to the range $k_{g}^{\max }$, for reasons which will be explained below. The values of the elements in gbest $s$ are determined by comparing the best performances of all the members of $i$ 's topological neighborhood, defined by indexes of some other population members, and assigning the best performer's index to the variable $g$. Thus gbest $g$ represents the best position found by any member of the neighborhood.

We also focused our work on the advantage of PSO (Particle Swarm Optimization) for the mutation process of GA (Genetic Algorithm), for improving the GA learning efficiency [5]. Euclidean distance is used for crossover to avoid local optimal solutions and to obtain fast running time for finding solutions. Using the conventional GA or PSO approach optimal solutions is obtained mostly with some initial differentiated data and there is a high possibility for obtaining local optimal solutions. The proposed approach uses data points with the longest Euclidean distance for crossover process to avoid such local optimization. The idea is to obtain global solutions by considering the entire search space (all the data points). Figure 1 shows a brief overview of GA-PSO structure applied for tuning some parameters for energy saving.

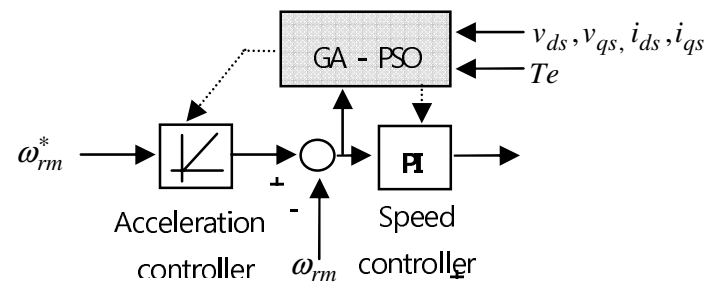

Figure 1. GA-PSO based energy saving control

\section{Genetic Algorithm -Bacterial Foraging Algorithm}

Search and optimal foraging based decision-making of animals can be used to engineering applications [7]. While performing social foraging an animal needs communication capabilities, it can gain advantages by essentially exploiting sensing capabilities of the group.

$$
\begin{aligned}
P_{c}(\phi) & =\sum_{i=1}^{N} P_{c c}^{i} \\
= & \sum_{i=1}^{N}\left[-L_{\text {atrract }} \exp \left(-\delta_{\text {atract }} \sum_{j=1}^{n}\left(\phi_{j}-\phi_{j}^{i}\right)^{2}\right)\right] \\
& +\sum_{i=1}^{N}\left[-K_{\text {repellant }} \exp \left(-\delta_{\text {repellant }} \sum_{j=1}^{n}\left(\phi_{j}-\phi_{j}^{i}\right)^{2}\right)\right],
\end{aligned}
$$

where $\phi=\left[\phi_{1, \ldots,} \phi_{p}\right]^{T}$ is a point on the optimization domain, $L_{\text {attract }}$ is the depth of the attractant released by the cell and $\delta_{\text {attract }}$ is a measure of the width of the attractant signal. $K_{\text {repellant }}=L_{\text {attract }}$ is the height of the repellant effect magnitude, and $\delta_{\text {attract }}$ is a measure of the width of the repellant. The expression means that its value does not depend on the nutrient concentration at position.

Below the method is presented in the form of an algorithm to search the optimal values of parameters. The transfer function of PID controller of AVR system is

$$
G(s)=k_{p}+\frac{k_{i}}{s}+k_{d} s
$$

and block diagram of AVR system is shown as Figure 2. The performance index of the control response is defined by: 


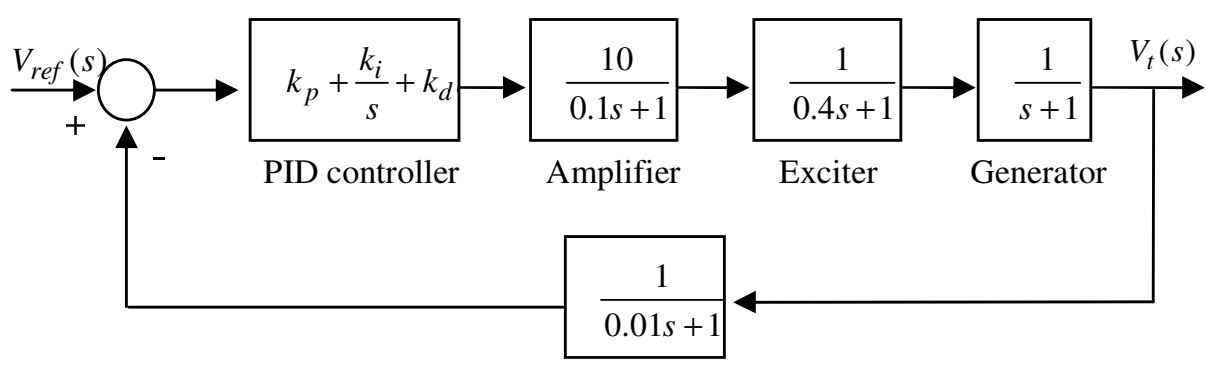

Sensor

Figure 2. Block diagram of an AVR system with a PID controller.

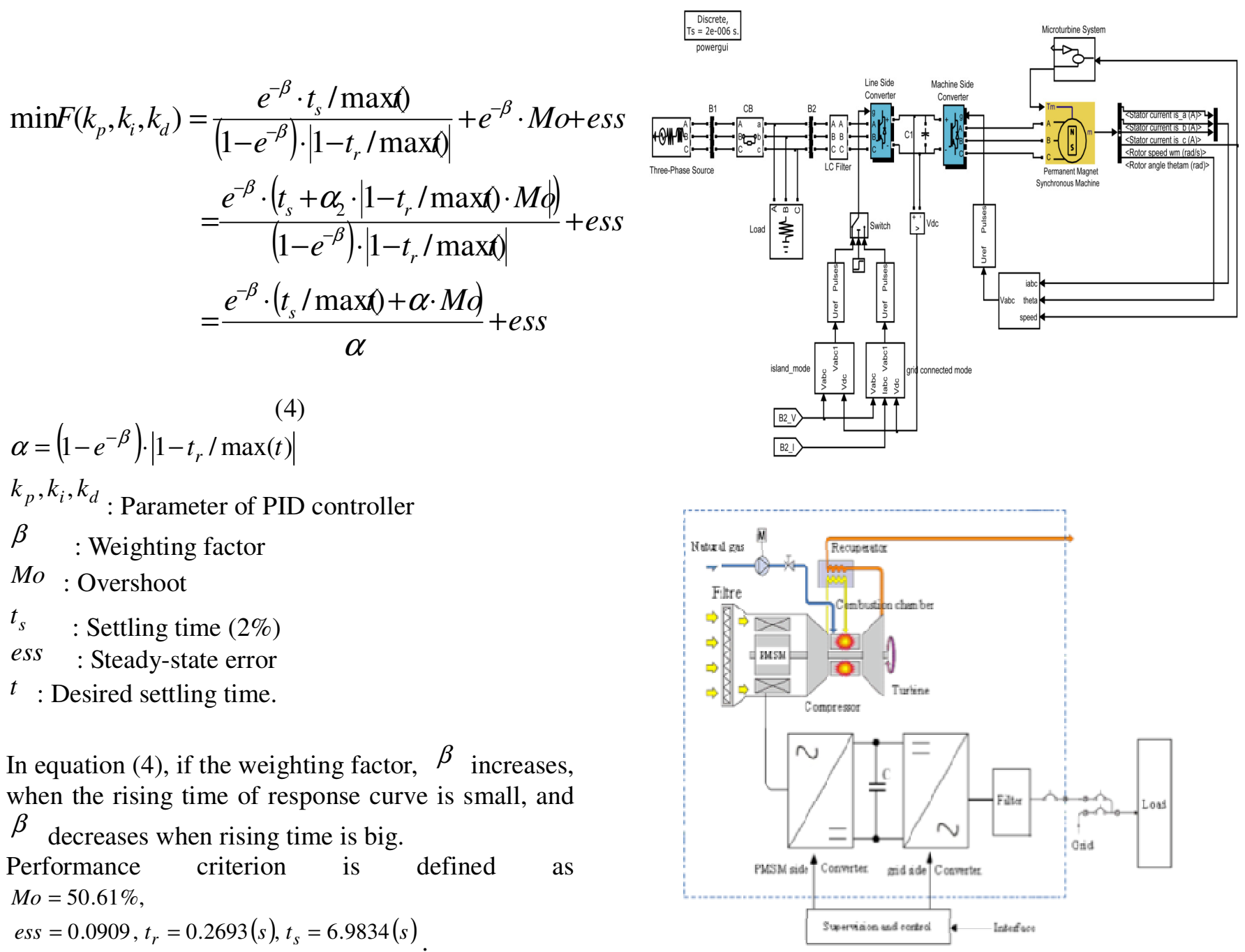

\section{Operation of Micro Turbine}

The turbine system is complicated with various components. So, there many control loops to control and optimal control algorithm should be applied to decouple to control efficiently.

Figure $3(\mathrm{a}, \mathrm{b})$ illustrates the micro turbine simulation block diagram.

Power Management Systems increase availability of network infrastructure by monitoring and controlling of power system for PC, IC, network, equipment, remotely. We measure input signal and out signal, then optimize for energy saving. 


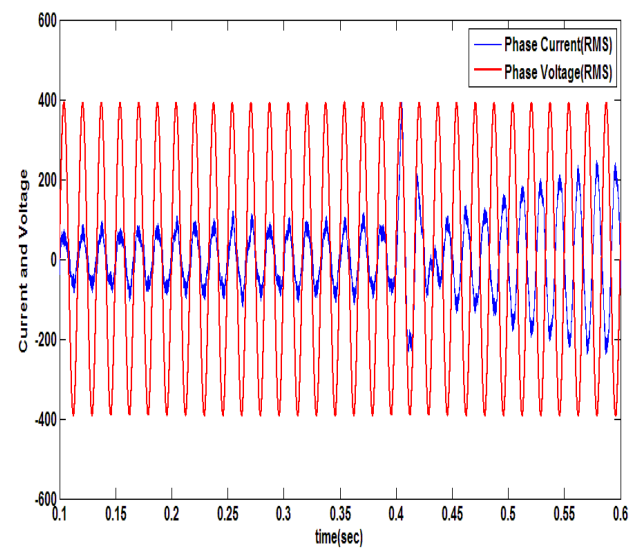

Figure 3(b). Simulation for micro turbine by intelligence system
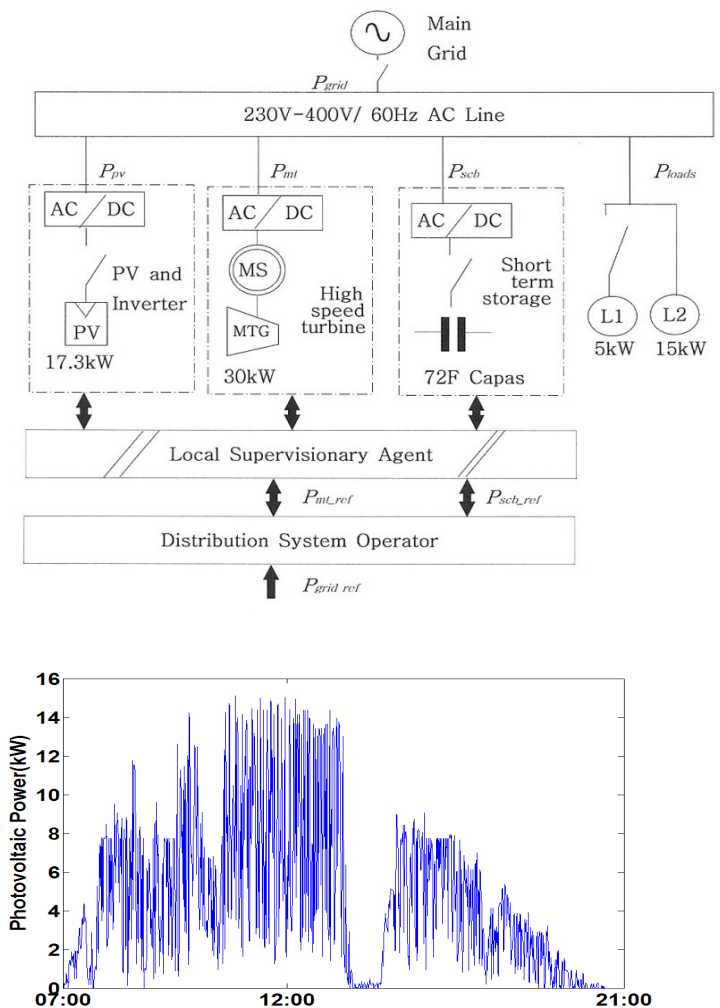

Figure 4. Power management structure for hybrid system MTG + PV + UBC

\section{Conclusions}

Energy saving and efficiently operating /control system is very important for green technology. The green energy technology refers to the application of knowledge for practical purposes. Therefore, the field of green technology encompasses a continuously evolving group of methods and materials, from techniques for generating energy to non-toxic cleaning products. Energy source reduction and saving is to reduce waste and pollution, developing alternatives is important to fossil fuel.

Green building is designed and operated for energy saving. For these ones, intelligent technology should be introduced in various for building. Green nanotechnology is also should introduced into intelligent technology for energy saving. For these purpose, GA-PSO and GA-BFO can be usefully applied for green technology effectively.

\section{Acknowledgements}

The work developed in this paper has been supported by the DAEDEOK INNOPLIS ("R\&D Hub Cluster project").

\section{References}

[1]D. Kim, A. Abraham, and J. Cho (2007), A hybrid genetic algorithm and bacterial foraging approach for global optimization," Information Sciences (177) :3918-3937

[2] M. Tripathy, S. Mishra, L. Lai, and Q. Zhang, "Transmission loss reduction based on facts and bacteria foraging algorithm," LNCS, vol. 4193, p. 222, 2006.

[3] W. O'brien, H. Browman, and B. Evans, "Search strategies of foraging animals," American Scientist, vol. 78, pp. 152-160, 1990.

[4] K. Passino, Biomimicry of bacterial foraging for distributed optimization andcontrol. IEEE Control Systems Magazine (22): 52-67.

[5] Dong Hwa Kim, Tokyo Institute of Technology Thesis, Genetic Algorithm Combined with Particle Swarm Optimization/Bacterial Foraging and Its Application to PID Controller Tuning, Tokyo, 2006.

[6] Dong Hwa Kim, "Intelligent tuning of AVR system using reference model based immune algorithm and PSO," IICAI2005 (LNCS-SCI), pp. 3402-3407, 2005.

[7] Dong Hwa Kim, Chae Hoon Cho, "A Biologically Inspired Intelligent PID Controller tuning For AVR System," IJCAS (SCI), Vol. 4, No. 5, 2006, Korea.

[8] Dong Hwa Kim, Jin Ill Park, "GA-PSO Based Vector Control For Indirect of Three Phase Induction Motor, Elsevier, Volume 7, pp. 601-611, 2007.

[11] Dong-Hwa Kim and Ajith Abraham, "Optimal Learning of Fuzzy Neural Network Using Artificial Immune Algorithm," Neural network world," 18(2) pp. 147-170, 2008.

[12] Dong Hwa Kim, Kaoro Hirota, "Vector Control for Loss Minimization of Induction Motor Using GA-PSO," Applied Soft Computing, pp. 16921702, 2008. 